\title{
Red cell distribution width and prognosis in acute heart failure: ready for prime time!
}

\author{
Roger Hullin $^{1}{ }^{1} \cdot$ Nicolas Barras $^{1} \cdot$ Tamila Abdurashidova $^{1} \cdot$ Pierre Monney $^{1} \cdot$ Julien Regamey $^{1}$
}

Received: 19 November 2018 / Accepted: 22 November 2018 / Published online: 13 December 2018

c) Società Italiana di Medicina Interna (SIMI) 2018

The prognosis of acute heart failure (AHF) remains generally but not uniformly poor with all-cause mortality (ACM) rising to $10-20 \%$ at $2-3$ months and $17-37 \%$ at 1 year. Moreover, almost $25 \%$ of patients are readmitted within 30 days, and up to $46 \%$ within 6 months after discharge from hospitalization for AHF. Many patients will die within the first few months after hospitalization for heart failure (HF), but most will not. Delay of discharge or more intensive post-discharge surveillance may improve the prognosis of AHF patients at high short-term risk of readmission or death, but this requires risk stratification of the individual patient already during the hospitalization. Most important, prediction should be easily applicable and adequate to make risk stratification of this high-risk population worthwhile $[1,2]$.

Biomarkers have been shown to improve prediction on top of clinical assessment in patients with chronic heart failure (HF), but the incremental predictive value of a single biomarker such as B-type natriuretic peptide or troponin I in AHF is small when added to a clinical evaluation [3]. Nevertheless, results from the Multinational Observational Cohort on Acute Heart Failure (MOCA) study suggest improved risk prediction if miscellaneous biomarkers are considered for assessment of different signaling pathways engaged in HF.

Red blood cells display physiological size heterogeneity in the adult, and the distribution of red cell distribution width (RDW) is a parameter routinely reported in complete blood cell counts. Increase of RDW has been associated with an higher risk for progression of cardiovascular disease, cancer and renal disease in multiple studies suggesting that this

Roger Hullin

roger.hullin@chuv.ch

1 Service de Cardiologie, Département Coeur-Vaisseaux, Centre Hospitalier Universitaire Vaudois, Université de Lausanne, BU44_07_2208, Rue du Bugnon 44, 1011 Lausanne, Switzerland parameter might also serve for prediction of prognosis in chronic HF.

In fact, RDW appears as the strongest predictor for adverse outcome among 36 laboratory values considered in the Candesartan in Heart Failure: Assessment of Reduction of Mortality and Morbidity (CHARM) study as demonstrated in the 2679 patients of North American Candesartan HF cohort. This association is further validated and confirmed in a replication set of 2410 matched patients from a local cohort. This predictive role of RDW in stable chronic $\mathrm{HF}$ is corroborated in a meta-analysis including a large number of smaller studies that investigated the role of RDW in HF. In addition, increased RDW is a predictor of incident HF and all-cause mortality in a post hoc analysis of the 4111 participants in CARE study (Cholesterol and Recurrent Events), which had included patients with stable coronary artery disease. Altogether, present evidence promotes the use of RDW for estimating prognosis in HF, and its application for prognostication of outcome in stable coronary artery disease.

In the past few years, various prospective cohort studies have investigated the role of RDW for risk assessment in patients with acute HF. Most important, all these studies show a consistent association of increased RDW at admission with all-cause mortality [4-9]. This association is maintained even when AHF patients are separated into a group with preserved left ventricular ejection fraction or reduced left ventricular ejection fraction $[5,9]$.

A number of studies further refine risk prediction in acute HF by RDW measurement at admission and discharge based on the hypothesis that clinical improvement from hospitalization for HF might have an impact on RDW. Increase of RDW between measure at admission and discharge is associated with an higher all-cause mortality in prospectively followed acute HF cohorts from South Korea $(n=261)$, Israel $(n=614)$ [4], Spain $(n=1702)$ [5], the US $(n=6414)$ [6], Japan $(n=229)$ [7], Switzerland $(n=402)$ [9], and in the Italian RE-AHF study $(n=451)$ [8]. The consistency of 
the association of RDW increase with all-cause mortality (HR 1.08-1.54) conjoined with the stability of this association across different ethnicities altogether provides a strong argument for recognition and broad application of this easily available parameter.

Improving 30-days readmission rate continues to be problematic for many hospitals, and more recently, a gender-specific 30-days readmission risk score has been derived in a HF cohort of the Intermountain Healthcare group $(n=6079)$ out of 182 inpatient data elements per patient. The components of the gender-specific inpatient HF risk score differ between males and females, and RDW was included only in the male inpatient HF score. Validation of the gender-specific inpatient risk score in another inpatient HF cohort $(n=5162)$ confirms applicability of the gender-specific inpatient scores [1]. For the moment it remains unclear whether the genderspecific relevance of RDW is related only to short-term prediction, or whether gender really plays a role. However, RDW is a component of another non-gender-specific risk score developed for prediction of 1-year all-cause mortality and rehospitalization after AHF related hospitalization [2]. Of note, in the latter study B-type natriuretic peptide is not related with the combined endpoint while, in contrast, RDW is so strongly related that it is entered into the risk score [2].

The question remains how to integrate the measure of the RDW into the clinical pathway of patients admitted for AHF. The "Recommendations on the pre-hospital and early hospital management of acute heart failure" by the European Society of Cardiology, the Society of Emergency Medicine and the Society of Academic Emergency Medicine provide helpful guidance for care of AHF within the initial 30-60 min, and observation for the subsequent 60-120 min. Thereafter, the guidelines recommend implementation of oral disease-modifying therapy in consideration of an individual patient's clinical presentation, while advice on risk stratification of the individual patient already defined during the hospitalization is missing.

On the basis of the current evidence, appraisal of the role of RDW for the discharge management of patients hospitalized for AHF is warranted. It is true that the association of RDW with non-cardiovascular disease could falsify the cardiovascular prognosis. However, anisocytosis can contribute to the progression of HF because in conditions of high-anisocytosis, red blood cells present a reduced deformability and decreased oxygen-carrier binding capacity, which can reduce tissue oxygenation in the myocardium and in peripheral tissues. While this suggests a direct impact of RDW on myocardial function, HF in itself may have effects on RDW. HF is associated with activation of both cell- and cytokine-mediated inflammatory pathways, which can induce bone marrow dysfunction with release of premature erythrocytes into the circulation. HF is furthermore related to nutritional deficiencies and local oxidative stress, which can likewise derange hematopoiesis. HF also promotes renal dysfunction resulting in anemia and anisocytosis [10]. In addition, older age is associated with anisocytosis as a result of multiple metabolic dysfunctions; moreover, advanced age is likewise a risk factor for heart failure. Last but not least, iron deficiency (ID) is another cause for anisocytosis, and ID is not only highly prevalent in chronic HF, but also in acute HF with a reported prevalence of $58-83 \%$. Correction of ID with intravenous ferric carboxymaltose is shown to decrease RDW in a subanalysis of the Ferinject Assessment in Patients with Iron Deficiency and Chronic Heart Failure (FAIR-HF) study. Whether ID correction during an AHF hospitalization can reduce mortality and readmission is currently being evaluated by the Study to Compare Ferric Carboxymaltose With Placebo in Patients With Acute Heart Failure and Iron Deficiency (AFFIRM-AHF) study (NCT02937454).

Altogether, current evidence suggests a pathophysiological concept of broad interaction between RDW and HF, which can explain the strong association between RDW and risk prediction in heart failure disease.

\section{Compliance with ethical standards}

Conflict of interest Authors declare that they have no conflict of interest.

Statement of human and animal rights This article does not contain any studies with human participants or animals performed by any of the authors.

Informed consent None.

\section{References}

1. Horne BD, Budge D, Masica AL, Savitz LA, Benuzillo J, Cantu G, Bradshaw A, Mc Cubey RO, Bair TL, Roberts CA, Rasmusson KD, Alharethi R, Kfoury AG, James BC, Lappé DL (2017) Early inpatient calculation of laboratory-based 30-day readmission risk scores empowers clinical risk modification during index hospitalization. Am Heart J 185:101-109

2. Xanthopoulos A, Giamouzis G, Tryposkiadis K, Paraskevopoulou E, Paraskevopoulou P, Karagiannis G, Patsilinakos S, Parissis J, Farmakis D, Butler J, Skoularigis J, Triposkiadis F (2017) A simple score for early risk stratification in acute heart failure. Int J Cardiol 230:248-254

3. Cleland JGF, Teerlink JR, Davison BE, Shoaib A, Metra M, Senger S, Milo O, Cotter G, Bourge RC, Parker JD, Jondeau G, Krum $\mathrm{H}$, O'Conner Torre-Amione G, van Veldhuisen D, McMurry JJ (2017) Measurement of troponin and natriuretic peptides shortly after admission in patients with heart failure-does it add useful prognostic information? An analysis of the value of endothelin receptor inhibition with tezosentan in acute heart failure studies (VERITAS). Eur J Heart Fail 19:739-747

4. Makhoul BF, Khourieh A, Kaplan M, Bahouth F, Aronson D, Azzam ZS (2013) Relation between changes in red cell 
distribution width and clinical outcomes in acute decompensated heart failure. Int J Cardiol 167:1412-1416

5. Núñez J, Núñez E, Rizopoulos D, Miñana G, Bodí V, Bondanza L, Husser O, Merlos P, Santas E, Pascual-Figal D, Chorro FJ, Sanchis J (2014) Red blood cell distribution width is longitudinally associated with mortality and anemia in heart failure patients. Circ J 78:410-418

6. Muhlestein JB, Lappe DL, Anderson JL, Muhlestein JB, Budge D, May HT, Bennett ST, Bair TL, Horne BD (2016) Both initial red cell distribution width (RDW) and change in RDW during heart failure hospitalization are associated with length of hospital stay and 30-day outcomes. Int J Lab Hematol 38:328-337

7. Uemura Y, Shibata R, Takemoto K, Uchikawa T, Koyasu M, Watanabe H, Mitsuda T, Miura A, Imai R, Watarai M, Murohara $\mathrm{T}$ (2016) Elevation of red blood cell distribution width during hospitalization predicts mortality in patients with acute decompensated heart failure. J Cardiol 67:268-273
8. Melchio R, Rinaldi G, Testa E, Giraudo A, Serraino C, Bracco C, Spadafora L, Falcetta A, Leccardi S, Silvestri A, Fenoglio L (2018) Red cell distribution width predicts mid-term prognosis in patients hospitalized with acute heart failure: the RDW in acute heart failure (RE-AHF) study. Intern Emerg Med. https://doi. org/10.1007/s11739-018-1958-Z

9. Sotiropoulos K, Yerly P, Monney P, Garnier A, Regamey J, Hugli O, Martin D, Metrich M, Antonietti JP, Hullin R (2016) Red cell distribution width and mortality in acute heart failure patients with preserved and reduced ejection fraction. ESC Heart Fail 3:198-204

10. Lippi G, Turcato G, Cervellin G, Sanchis-Gomar F (2018) Red blood cell distribution width in heart failure: a narrative review. World J Cardiol 10:6-14 